\title{
Wind Field of a Nonmesocyclone Anticyclonic Tornado Crossing the Hong Kong International Airport
}

\author{
Karen A. Kosiba, ${ }^{1}$ Paul Robinson, ${ }^{1}$ P. W. Chan, ${ }^{2}$ and Joshua Wurman ${ }^{1}$ \\ ${ }^{1}$ Center for Severe Weather Research, 1945 Vassar Circle, Boulder, CO 80305, USA \\ ${ }^{2}$ Hong Kong Observatory, 134A Nathan Road, Hong Kong \\ Correspondence should be addressed to Karen A. Kosiba; kakosiba@cswr.org
}

Received 19 October 2013; Revised 24 December 2013; Accepted 31 December 2013; Published 2 March 2014

Academic Editor: Sven-Erik Gryning

Copyright (C) 2014 Karen A. Kosiba et al. This is an open access article distributed under the Creative Commons Attribution License, which permits unrestricted use, distribution, and reproduction in any medium, provided the original work is properly cited.

\begin{abstract}
A nonmesocyclone tornado traversed the Hong Kong International Airport on September 6, 2004 directly impacting a surface weather station. This allowed for 1-second 10-meter above ground level (AGL) wind observations through the core of the tornado. Integration of these 10-meter AGL wind data with Ground-Based Velocity Track (GBVTD) wind retrievals derived from LIDAR data provided a time history of the three-dimensional wind field of the tornado. These data indicate a progressive decrease in radial inflow with time and little to no radial inflow near the time the tornado crosses the surface weather station. Anemometer observations suggest that the tangential winds approximate a modified-Rankine vortex outside the radius of maximum winds, suggesting that frictionally induced radial inflow was confined below $10 \mathrm{~m}$ AGL. The radial-height distribution of angular momentum depicts an increase in low-level angular momentum just prior to the tornado reaching its maximum intensity.
\end{abstract}

\section{Introduction}

Although fine-scale observations of tornado structure have occurred more frequently in recent years with the proliferation of mobile radars and focused field experiments (e.g., [17]) with few exceptions (e.g., [8-13]) low-level observations, below 15-20 $\mathrm{m}$ above ground level (AGL), are comparatively rare. Even mobile radar observations near the surface are complicated by intervening ground clutter and degraded resolution and increased observing height with increased distance from the tornado [14]. In situ observations also are difficult to obtain due to the inherent dangers of placing instrumentation in the path of violent and often debris-laden winds. All observations of tornadoes are, of course, limited by the crude predictability of formation and track, as well as the short temporal and small spatial scales characteristic of the phenomenon. However, characterizing the near-surface winds in a tornado is critical for understanding how these winds cause damage and how near-surface wind velocities relate to those more frequently observed aloft.

The tornado wind field is commonly partitioned into the following different flow regimes: (i) the swirling boundary layer, (ii) the core region, (iii) the corner region, and (iv) the upper flow (e.g., see reviews by [15-17]). Briefly, the boundary layer, which forms as a result of the rotating flow's interaction with the underlying surface, feeds the core flow by way of the corner flow region. Of particular interest to this study is the relationship between the core flow and boundary layer winds. Surface friction results in an imbalance between the centrifugal and radial pressure gradient forces, which acts to increase near-surface inflow. This leads to an increase in the tangential velocities in the mid and upper portions of the boundary layer. Additionally, large eddy simulations [18] suggest that turbulent transport in the boundary can increase tangential winds by up to $30 \%$ just above the surface. Observations by Wurman et al. [9] document little reduction in wind speed between measurements taken at $3 \mathrm{~m}$ above ground level (AGL) and those taken at $18 \mathrm{~m}$ AGL, possibly suggesting that $3 \mathrm{~m}$ AGL is near the top of the boundary layer in that case. Substantial radial inflow at $3 \mathrm{~m}$ AGL was documented in a strong tornado by Wurman et al. [12], but due to the available data, it was difficult to determine whether the total winds increased with height or were constant between $3 \mathrm{~m}$ AGL and $20 \mathrm{~m}$ AGL; therefore the depth of the boundary layer may have been near $3 \mathrm{~m}$ AGL or much higher. Integrated low-level radar data and in situ observations in 


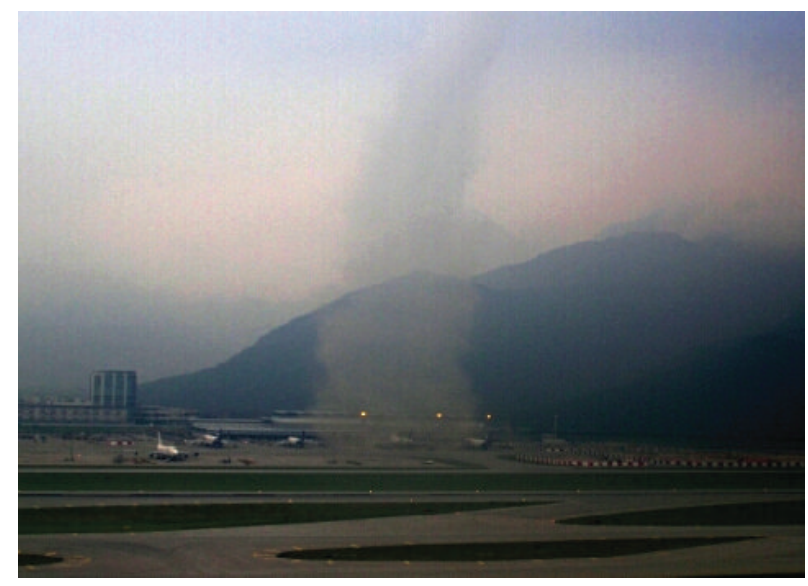

(a)

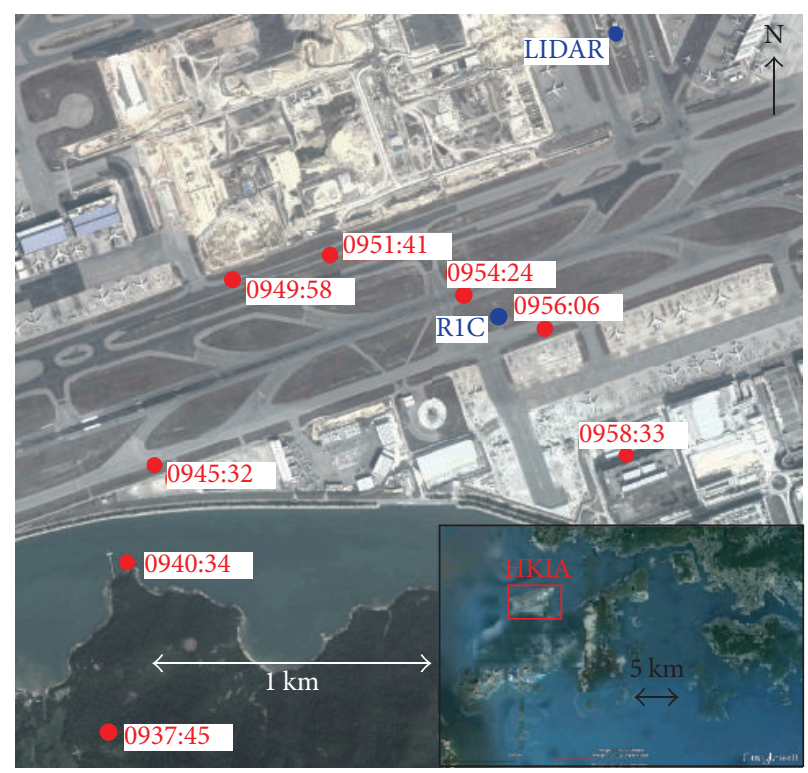

(b)

Figure 1: (a) Photograph of the tornado taken from the control tower (located near the LIDAR) at the Hong Kong International Airport at approximately 0957 UTC. View is towards the south southwest. (b) Location of the tornado center (red dot), as determined from the LIDAR data, at the times indicated in red. The location of the LIDAR and the surface weather station RC1 are indicated in blue.

a weak tornado [13] depicted a weak, shallow $(\sim 10 \mathrm{~m})$ inflow layer with the strongest winds occurring within $5 \mathrm{~m}$ AGL. An accurate assessment of the boundary layer depth in tornadoes is needed to fully understand and model the wind distribution with height.

\section{Data and Methodology}

On September 6, 2004, an anticyclonic tornado formed behind the leading edge of a thunderstorm gust front and crossed the southwestern portion of the Chek-Lap-Kok International Airport in Hong Kong, China (Figure 1). Observations were available from several platforms, including a Terminal Doppler Weather Radar (TDWR), a LIDAR, and a surface weather station. At 0946 UTC (all times hereafter are in UTC), TDWR and LIDAR data depict four distinct anticyclonic circulations along the gust front (Figure 2(a)), but only circulation number 3 persisted and briefly intensified to marginally tornado strength (Consistent with the Wurman and Kosiba [19] classification of a marginal tornado.) (Figures 2(b) and 3). One of the airport surface weather stations (R1C) was directly impacted by the tornado, thereby providing rarely obtained in situ observations of the nearsurface winds in a tornado. These surface data combined with proximal and contemporaneous LIDAR observations allow for the reconstruction of the two-dimensional time history of the winds throughout the lifetime of the tornado.

The LIDAR was located $50 \mathrm{~m}$ AGL on the roof of an airport building. LIDAR data comprised horizontal sector scans ranging in elevation from 0.0 to 4.5 degrees. This scan strategy was repeated approximately every two minutes. Range gates were every $105 \mathrm{~m}$ and azimuthal sampling was in 1-degree increments. At the time of closest approach, the tornado was $1.1 \mathrm{~km}$ from the LIDAR, yielding an azimuthal spacing of $20 \mathrm{~m}$. The surface weather station (R1C hereafter) used in this study is located at a distance of $1.09 \mathrm{~km}$ and an azimuth of 202.6 degrees from the LIDAR. The anemometer was located at a height of $10 \mathrm{~m}$ AGL and winds (direction and speed) were sampled at 1-second intervals. The error associated with the location of a given LIDAR volume measurement is approximately one-half the sample volume, or $53 \mathrm{~m}$ in range and $9 \mathrm{~m}$ in azimuth (at the location of the tornado).

The Ground-Based Velocity Track (GBVTD) technique $[5,6,11,13,20,21]$ was used to retrieve the three-dimensional axisymmetric wind components from the LIDAR data during the life cycle of the tornado, including the time of the tornado's closest approach to R1C. Before the GBVTD technique was applied, the data were interpolated to a Cartesian grid using a Barnes objective analysis scheme [22]. Since LIDAR sampling was mismatched in range $(105 \mathrm{~m})$ and azimuth $(\sim 30 \mathrm{~m})$ at the location of the tornado, a smoothing parameter $(\kappa)$ of $0.005402 \mathrm{~km}^{2}$ was applied to the data, which was slightly less than the value recommended by Pauley and $\mathrm{Wu}$ [23] and Koch et al. [24] (it is generally recommended that $\kappa=(1.33 \Delta)^{2}$, where $\Delta$ is the coarsest data spacing, to mitigate the introduction of noise into the analysis, but since the LIDAR azimuthal data spacing is much finer than the range data spacing, a less conservative value of $\kappa\left[=(0.7 \Delta)^{2}\right]$ was used to preserve the tangential velocities, which were well sampled in azimuth), and grid spacing was $40 \mathrm{~m}$ in the horizontal and vertical. Analyses were only conducted at times when volumetric data were available. Also, due to the relatively small size of the core (core size is defined as the distance between total wind maxima) in comparison to LIDAR sampling, the radial wind component (due to 
0946:19

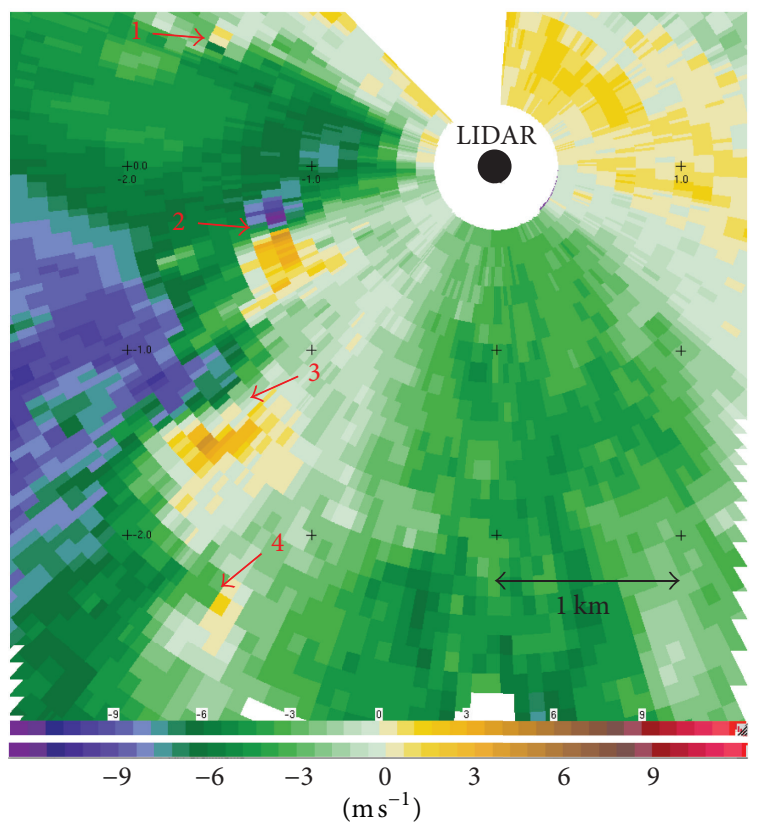

(a)

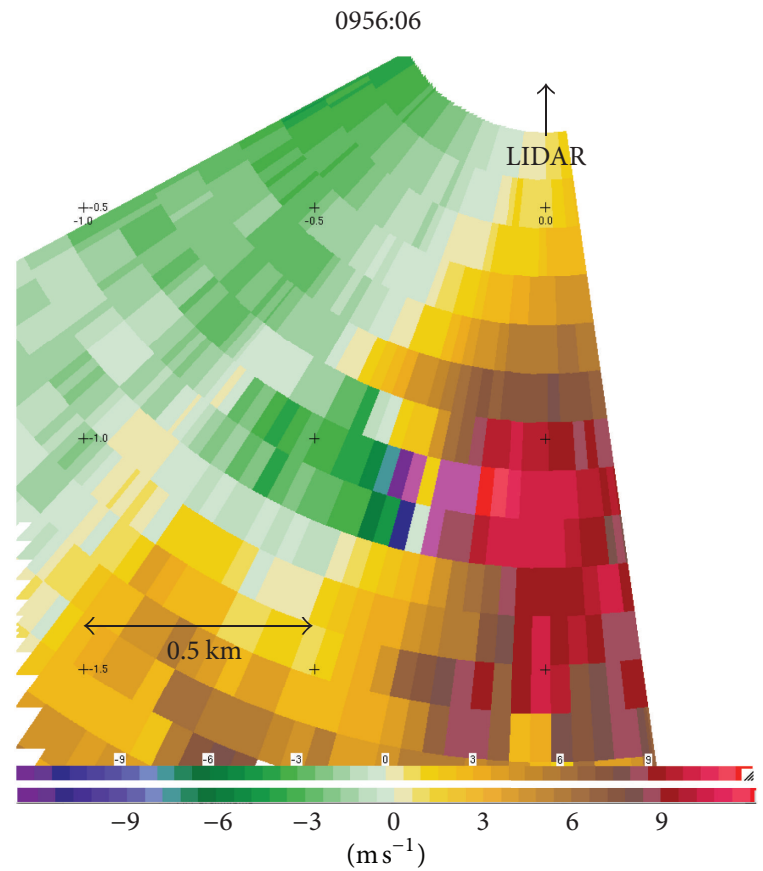

(b)

FIgURE 2: (a) LIDAR Doppler velocity at 0946:19, showing four weak circulations behind the leading edge of the gust front (not shown). Circulation number 3 becomes the tornado documented in this paper, while the other circulations do not persist. (b) LIDAR Doppler velocity at 0956:06, which is the closest scan to the time when the surface station was impacted by the tornado. Colors represent Doppler velocity with scale along the bottom in $\mathrm{m} \mathrm{s}^{-1}$.

LIDAR sampling geometry and parameters, the tangential winds were better resolved than the radial winds since the azimuthal sampling was approximately $30 \mathrm{~m}$ at the location of the tornado, whereas the radial sampling was $105 \mathrm{~m}$ ) was poorly resolved at and inside the radius of maximum winds (RMW) precluding an analysis of vortex structure at those locations.

\section{Results}

The intensity and radius of the tornado, as deduced from LIDAR observations, are depicted in Figure 3. The tornado gradually increases in strength from 0940:34 until 0956:06 to a maximum delta- $\mathrm{V}$ of $36 \mathrm{~m} \mathrm{~s}^{-1}$ and then decreases from 0956:06 to 0958:33. Correspondingly, the RMW decreases from $140 \mathrm{~m}$ to $30 \mathrm{~m}$ between 0940:34 and 0956:06 and then increases thereafter. Wurman and Kosiba [19] have documented short-lived tornadoes of similar strength (MTs; marginal tornadoes) and Tanamachi et al. [21] have analyzed the two-dimensional structure of MTs that occurred during the Second Verification of the Origins of Rotation Experiment [7]. Based on the LIDAR observations, the tornado was near peak intensity as it crossed weather station R1C at 0955:30. A photograph (Figure 1(a)) taken near the time the tornado traversed the weather station depicts a visible funnel cloud extending to the surface.

Since the core flow of the tornado crossed over the surface weather station, a transect of the near-surface tornado

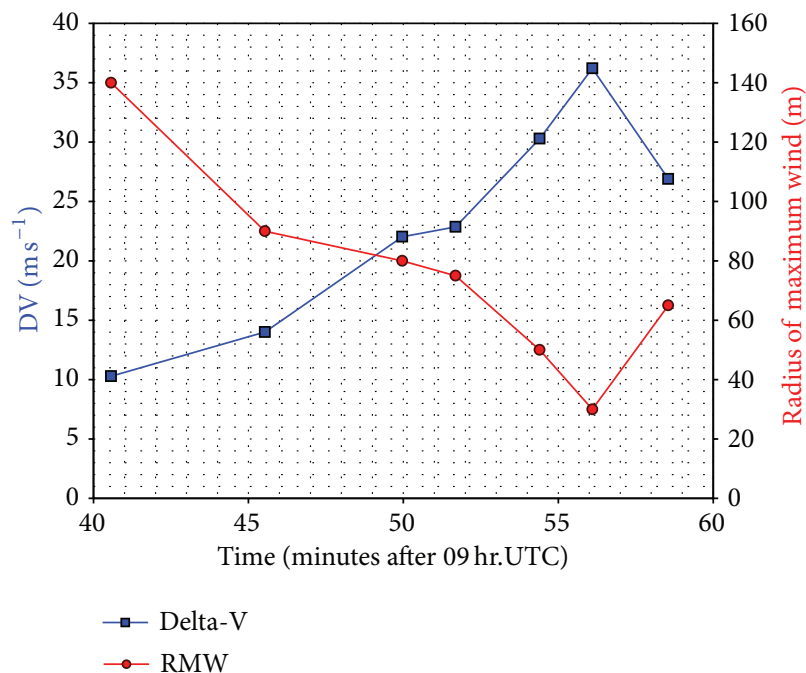

Figure 3: Time history of delta-V in $\mathrm{m} \mathrm{s}^{-1}$ (blue line) and the radius of maximum wind in $\mathrm{m}$ (red line) as determined from the LIDAR data.

core winds was obtained (Figure 4). Tornado motion was determined from the LIDAR data and then subtracted from the surface observations to obtain the tornado-relative winds. These winds were then compared to an idealized modifiedRankine vortex model [25] in order to ascertain information regarding the probable wind structure of the vortex. A Rankine vortex predicts the tangential winds $\left(V_{t}\right)$ as a function of 


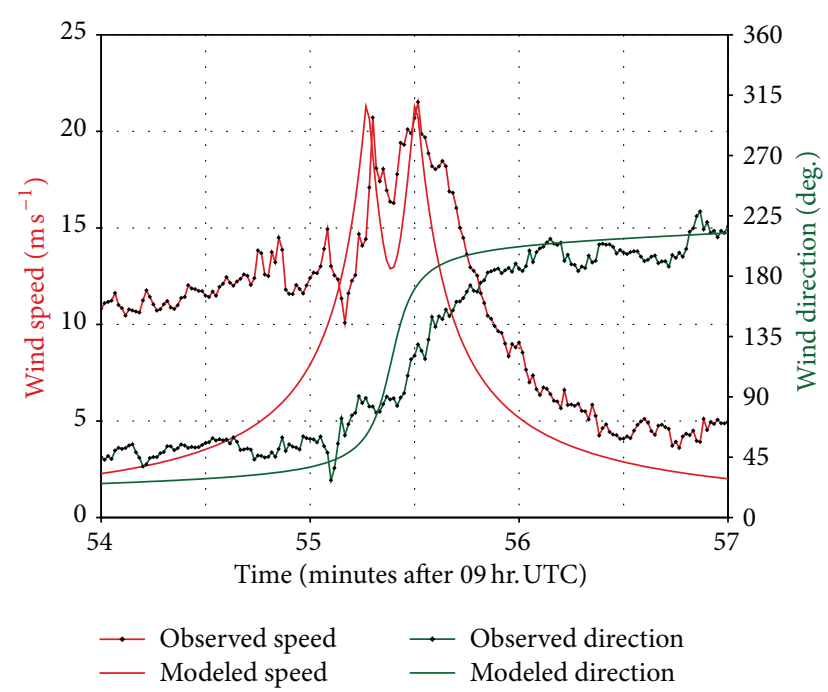

FIgURE 4: Tornado-relative wind speed in $\mathrm{m} \mathrm{s}^{-1}$ (red line with black dots) and direction in degrees (green line with black dots) from surface weather station R1C at 1-second intervals during the passage of the tornado. Best-fit Rankine tangential wind speed (solid red line) and direction (solid green line) profiles are shown.

radius $(r)$ by assuming potential flow outside and solid body rotation inside the core radius $\left(R_{0}\right)\left(V_{t} \sim r^{-\alpha}\right.$ for $r>R_{0} ; V_{t} \sim$ $r^{\alpha}$ for $r<R_{0}$, where $\alpha=1$ for a Rankine vortex and $\alpha<1$ for a modified-Rankine vortex). Previous studies of the tangential winds in tornadoes $[2,5,7,8,10,11]$ and in hurricanes [26] suggest that the decay exponent $(\alpha)$ approaches 0.5 , implying a loss of fluid momentum as the air spirals inward.

Anemometer data were fit to a modified-Rankine vortex profile between 0955:38 and 0956:31 as the tornado core passed over the weather station. The Rankine parameters $\left(R_{0}\right.$, $V_{0}$ [velocity at the core radius], and $\alpha$ ) were chosen based on minimization of the least squares error. Contrary to previous findings, the best fit was achieved with a decay exponent of one, perhaps suggesting that frictionally induced inflow near the surface is transporting higher angular momentum air inwards [27]. Radial profiles of the tangential winds constructed from quasi-contemporaneous LIDAR data, approximately $40 \mathrm{~m}$ above the anemometer, suggested that $\alpha<1$, with values ranging from 0.7 to 0.9 . It is possible that $\alpha$ varies with height, but the coarseness of the LIDAR data samples compared to the anemometer data may also account for this discrepancy. Sensitivity of the fit as determined through a least squares minimization to varying amounts of radial wind $\left(V_{r}\right)$ revealed little to no $V_{r}$, indicating that flow was primarily tangential at this time.

Analysis of quasi-contemporaneous LIDAR and photographic data taken near the time of the tornado's encounter with R1C (Figure 1(a)) were used to estimate an $R_{0}$ of $30 \mathrm{~m}( \pm 5 \mathrm{~m})$. Based on interpolation of the tornado center location as determined by LIDAR observations (the center of the tornado relative to the LIDAR was determined by a third-order polynomial interpolation between LIDAR observations), the $R_{0}$ value suggests that the anemometer observations were at or very near to the core radius. This,

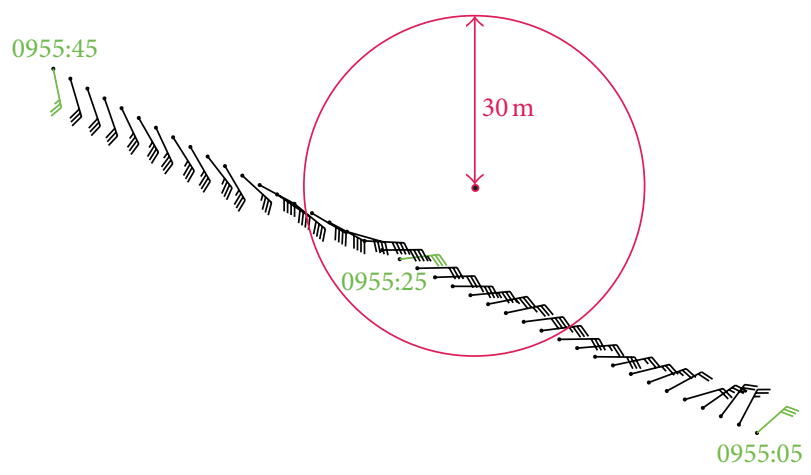

FIGURE 5: Time-to-distance conversion of tornado-relative winds as observed at surface station R1C, resulting in a transect through the core flow region of the tornado. The black dot indicates location of the tornado center and the circle denotes the radius of maximum winds. Time along the wind-barb path advances from 0955:05 (lower right) to 0955:45 (upper left) at one-second intervals. Select times are shown in purple. The short (long) wind barb represents $2.5 \mathrm{~m} \mathrm{~s}^{-1}$ $\left(5 \mathrm{~m} \mathrm{~s}^{-1}\right)$.

along with the tornado-relative wind directions derived from the anemometer observations, suggests little to no deviation of the winds from a purely tangential flow (Figure 5), which is consistent with the derived modified-Rankine vortex profile. The lack of radial inflow near the surface is in contrast to the findings of Wurman et al. [12], who found significant radial inflow within 3 meters of the surface in a strong tornado, but is similar to the findings of Kosiba and Wurman [13], who found that the radial inflow substantially decreased above the surface boundary layer. It is possible that inflow only occurs within a very shallow region near the surface in weak tornadoes and that the current 10-meter observations were above the inflow layer. It is also possible that the inflow layer does extend to $10 \mathrm{~m}$ AGL but that the inflow is asymmetric and thus was not observed by the fixed-location anemometer observations, similar to the tornadic structure proposed in Wurman [28].

GBVTD analyses (based on visual observations, dust was likely the primary scatterer type; the results were relatively insensitive to the inclusion of a small bias $(4 \mathrm{~m} / \mathrm{s}$ ) to approximate the effects of debris centrifuging) were available approximately every 5 minutes (Figures 6(a)-6(e)). Early analyses, at 0941, reveal a weak vortex, with inflow turning upward as it approaches the RMW. By the next analysis time, 0945, near the RMW, there is a suggestion of a downdraft aloft $(200 \mathrm{~m}$ to $300 \mathrm{~m} \mathrm{AGL}$ ), which does not persist through the subsequent 0950 and 0954 analyses. The last analysis time, 0958, suggests a downdraft may have redeveloped and extended to the surface. From 0941 to 0958 , the tangential velocities continually increase in magnitude. Interestingly, the maximum tangential velocities extend through the depth of the domain at 0954 but are confined to the surface at 0958. Soon after 0958, the tornado dissipates. The angular momentum distribution suggests that as the tornado is intensifying (Figure 6(c)), increased angular momentum is being imparted to the circulation near the surface. The importance of this low-level angular flux in tornado intensification has been underscored 


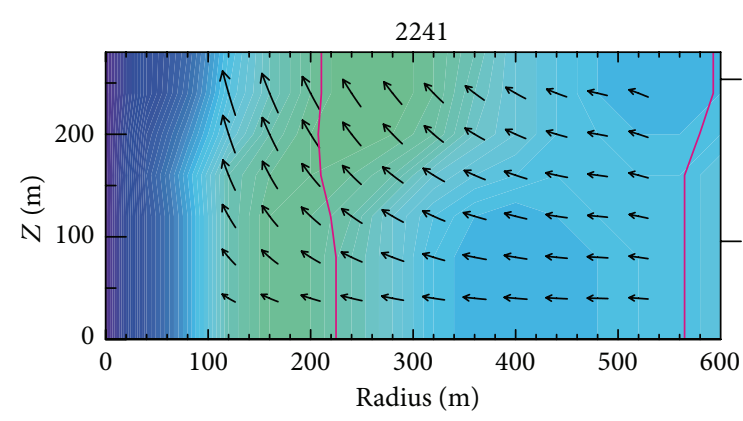

(a)

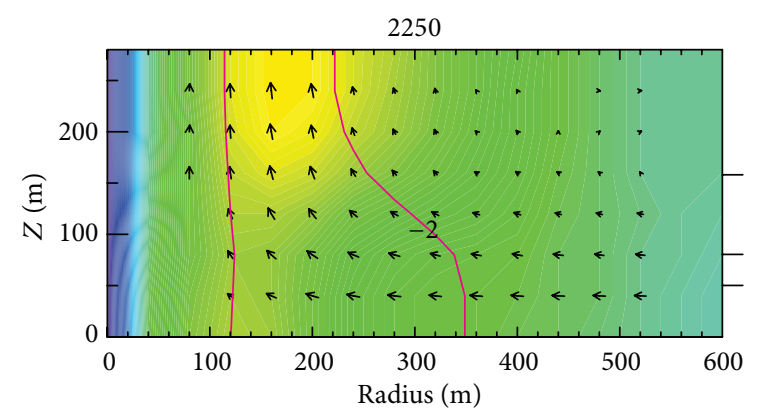

(c)

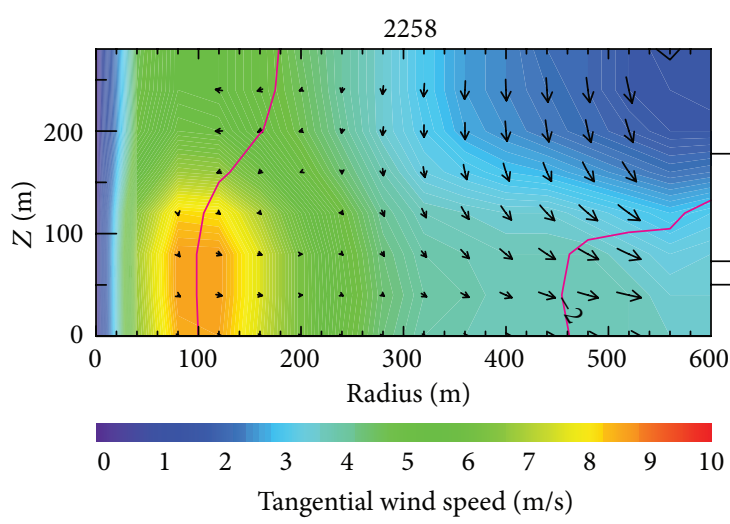

Reference vector

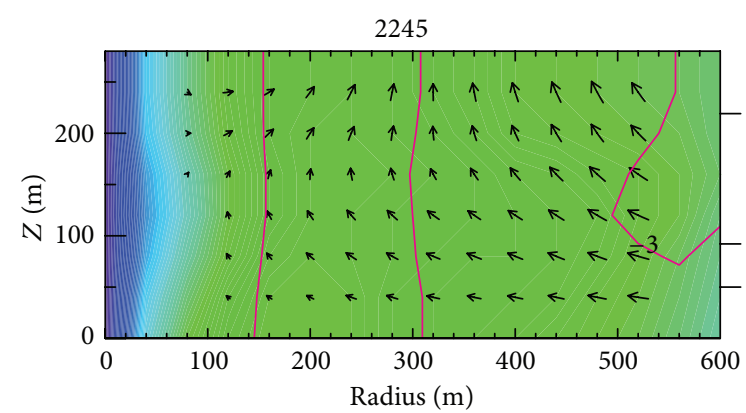

(b)

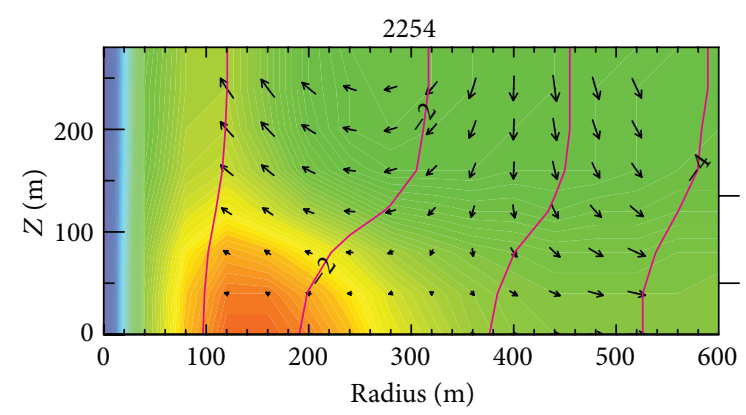

(d)

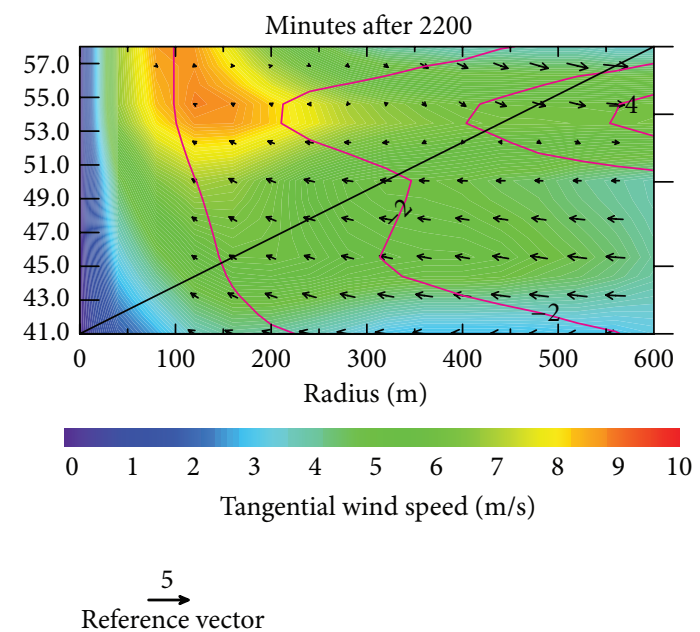

(f)

FIGURE 6: GBVTD retrieval of the three-dimensional tornado-relative axisymmetric winds for (a) 0941, (b) 0945, (c) 0950, (d) 0954, and (e) 0958. The horizontal axis indicates distance (in meters) from tornado center and the left (right) vertical axis indicates height above ground level of the GBVTD analysis (LIDAR observation). A Hövmoller diagram of the GBVTD winds at $60 \mathrm{~m}$ AGL is shown in (f). Time is on the vertical axis and radial distance from tornado center is on the $y$-axis. Vectors represent the radial-vertical winds $\left(\mathrm{m} \mathrm{s}^{-1}\right)$, color contours depict the tangential winds $\left(\mathrm{m} \mathrm{s}^{-1}\right)$, and the line contours are of angular momentum $\left(\mathrm{kg} \mathrm{km} \mathrm{m}^{-2} \mathrm{~s}^{-1}\right)$. Radial inflow decreases outside of the radius of maximum winds with time. Low-level angular momentum increases at 0954, just prior to maximum tornado intensity.

in numerical simulations (e.g., [27]) and similar angular momentum profiles during tornado intensification have been observed in other axisymmetric three-dimensional analyses of tornadoes (e.g., [10, 11, 13]). A Hövmoller diagram constructed for the two-dimensional winds at $60 \mathrm{~m} \mathrm{AGL}$ (Figure 6(f)) reveals a progressive decrease in radial inflow with time and an increase in angular momentum, just prior to observed maximum tangential winds.
In order to compare the derived two-dimensional structure of the winds near the surface with the winds aloft, the GBVTD technique was applied to the 0.0 degree LIDAR data at 0956:06, which was the closest scan in time when the tornado traversed the surface station, to derive the axisymmetric $V_{t}$ and $V_{r}$. Results depict weak, inflow $(\sim 1 \mathrm{~m} / \mathrm{s})$ for $110 \mathrm{~m} \leq r \leq 250 \mathrm{~m}$ and no inflow for $r \leq 80 \mathrm{~m}$. The tangential winds reached a maximum value of $17 \mathrm{~m} \mathrm{~s}^{-1}$ 
at a radius of $50 \mathrm{~m}$, consistent with the maximum tornadorelative wind speed of $21.5 \mathrm{~m} \mathrm{~s}^{-1}$ observed at R1C. There was no appreciable change in the tangential and radial wind speeds between the 10-meter AGL anemometer observations and the LIDAR data at $50 \mathrm{~m}$ AGL.

\section{Conclusions}

The three-dimensional wind field of an anticyclonic tornado that crossed the Hong Kong International Airport was deduced from 10-meter anemometer observations and LIDAR data at and above $50 \mathrm{~m}$ AGL. Three-dimensional GBVTD analyses of the LIDAR data revealed a decrease in radial inflow outside of the core radius with time. Wind speed and direction measurements from the anemometer data were fit to an idealized modified-Rankine vortex profile using a core radius of $30 \mathrm{~m}$ and maximum tangential wind speed of $22 \mathrm{~m} \mathrm{~s}^{-1}$. The data suggest that the edge of the inner core flow of the tornado passed almost directly over the anemometer and there was little to no inflow at 10 meters AGL. GBVTD analysis of the LIDAR winds also indicated a vortex consisting of almost purely tangential flow. This finding suggests that the frictional inflow layer is below 10 meters AGL but does not exclude the possibility that the inflow is asymmetric and unobserved by the available instrumentation. More nearsurface observations and vertical profiles of the low-level winds are needed to evaluate these hypotheses.

\section{Conflict of Interests}

The authors declare that there is no conflict of interests regarding the publication of this paper.

\section{Acknowledgment}

National Science Foundation Grant AGS-1211132 supported this work.

\section{References}

[1] J. Wurman, J. M. Straka, and E. N. Rasmussen, "Fine-scale Doppler radar observations of tornadoes," Science, vol. 272, no. 5269, pp. 1774-1777, 1996.

[2] J. Wurman and S. Gill, "Finescale radar observations of the Dimmitt, Texas (2 June 1995), tornado," Monthly Weather Review, vol. 128, no. 7, pp. 2135-2164, 2000.

[3] H. B. Bluestein, W.-C. Lee, M. Bell, C. C. Weiss, and A. L. Pazmany, "Mobile Doppler radar observations of a tornado in a supercell near Bassett, Nebraska, on 5 June 1999. Part II: tornado-vortex structure," Monthly Weather Review, vol. 131, no. 12, pp. 2968-2984, 2003.

[4] H. B. Bluestein, C. C. Weiss, and A. L. Pazmany, "The vertical structure of a tornado: high-resolution, W-band, Doppler radar observations near Happy, Texas on 5 May 2002," Monthly Weather Review, vol. 132, no. 10, pp. 2325-2337, 2004.

[5] W.-C. Lee and J. Wurman, "Diagnosed three-dimensional axisymmetric structure of the Mulhall tornado on 3 May 1999," Journal of the Atmospheric Sciences, vol. 62, no. 7, pp. 2373-2393, 2005.
[6] R. L. Tanamachi, H. B. Bluestein, W.-C. Lee, M. Bell, and A. Pazmany, "Ground-Based Velocity Track Display (GBVTD) analysis of W-band Doppler radar data in a tornado near Stockton, Kansas, on 15 May 1999," Monthly Weather Review, vol. 135, no. 3, pp. 783-800, 2007.

[7] J. Wurman, D. Dowell, Y. Richardson et al., "The second verification of the origins of rotation in tornadoes experiment: VORTEX2," Bulletin of the American Meteorological Society, vol. 93, no. 8, pp. 1147-1170, 2012.

[8] J. Wurman and C. R. Alexander, "The 30 May 1998 Spencer, South Dakota, storm. Part II: comparison of observed damage and radar-derived winds in the tornadoes," Monthly Weather Review, vol. 133, no. 1, pp. 97-119, 2005.

[9] J. Wurman, C. Alexander, P. Robinson, and Y. Richardson, "Low-level winds in tornadoes and potential catastrophic tornado impacts in urban areas," Bulletin of the American Meteorological Society, vol. 88, no. 1, pp. 31-46, 2007.

[10] K. A. Kosiba, R. J. Trapp, and J. Wurman, "An analysis of the axisymmetric three-dimensional low level wind field in a tornado using mobile radar observations," Geophysical Research Letters, vol. 35, no. 5, Article ID L05805, 2008.

[11] K. A. Kosiba and J. Wurman, "The three-dimensional axisymmetric wind field structure of the Spencer, South Dakota, 1998 tornado," Journal of the Atmospheric Sciences, vol. 67, no. 9, pp. 3074-3083, 2010.

[12] J. Wurman, K. A. Kosiba, and P. Robinson, "In-Situ, Doppler radar and video observations of the interior structure of a tornado and wind-damage relationship," Bulletin of the American Meteorological Society, vol. 94, no. 6, pp. 835-846, 2013.

[13] K. A. Kosiba and J. Wurman, "The three-dimensional structure and evolution of a tornado boundary layer," Weather and Forecasting, vol. 28, no. 6, pp. 1552-1561, 2013.

[14] C. R. Alexander and J. Wurman, "Comparison between DOW observed tornadoes and parent mesocyclones observed by WSR-88Ds," in Proceedings of the 22nd Conference on Severe Local Storms, American Meteorological Society, Hyannis, Mass, USA, 2004.

[15] W. S. Lewellen, "Assessment of knowledge and implications for man," in Proceedings of the Symposium on Tornadoes, pp. 107143, Texas Tech University, Lubbock, Tex, USA, 1976.

[16] W. S. Lewellen, “Tornado vortex theory", in The Tornado: Its Structure, Dynamics, Predictions and Hazards, C. Church, D. Burgess, C. Doswell, and R. Davies-Jone, Eds., vol. 79 of Geophysical Monograph, pp. 19-39, American Geophysical Union, 1993.

[17] R. P. Davies-Jones, R. J. Trapp, and H. B. Bluestein, “Tornadoes and tornadic storms," in Severe Convective Storms, vol. 50 of Meteorological Monographs, pp. 167-222, 2001.

[18] W. S. Lewellen, D. C. Lewellen, and R. I. Sykes, "Large-eddy simulation of a tornado's interaction with the surface," Journal of the Atmospheric Sciences, vol. 54, no. 5, pp. 581-605, 1997.

[19] J. Wurman and K. A. Kosiba, "Fine-scale radar observations of tornado and mesocyclone structures," Weather and Forecasting, vol. 28, no. 5, pp. 1157-1174, 2013.

[20] W.-C. Lee, B. J.-D. Jou, P.-L. Chang, and S.-M. Deng, “Tropical cyclone kinematic structure retrieved from single-doppler radar observations. Part I: interpretation of Doppler velocity patterns and the GBVTD technique," Monthly Weather Review, vol. 127, no. 10, pp. 2419-2439, 1999.

[21] R. L. Tanamachi, H. B. Bluestein, M. Xue et al., "Near-surface vortex structure in a tornado and in a sub-tornado-strength, 
convective-storm vortex observed by a mobile, W-band radar during VORTEX2," Monthly Weather Review, vol. 141, no. 11, pp. 3661-3690, 2013.

[22] S. L. Barnes, "A technique for maximizing details in numerical weather map analysis," Journal of Applied Meteorology, vol. 3, no. 4, pp. 396-409, 1964.

[23] P. M. Pauley and X. Wu, "The theoretical, discrete, and actual response of the Barnes objective analysis scheme for one- and two-dimensional fields," Monthly Weather Review, vol. 118, no. 5, pp. 1145-1163, 1990.

[24] S. E. Koch, M. Desjardins, and P. J. Kocin, "An interactive Barnes objective map analysis scheme for use with satellite and conventional data," Journal of Climate \& Applied Meteorology, vol. 22, no. 9, pp. 1487-1503, 1983.

[25] W. J. Rankine, A Manual of Applied Mechanics, Charles Griffin, London, UK, 1982.

[26] K. J. Mallen, M. T. Montgomery, and B. Wang, "Reexamining the near-core radial structure of the tropical cyclone primary circulation: implications for vortex resiliency," Journal of the Atmospheric Sciences, vol. 62, no. 2, pp. 408-425, 2005.

[27] D. C. Lewellen, W. S. Lewellen, and J. Xia, "The influence of a local swirl ratio on tornado intensification near the surface," Journal of the Atmospheric Sciences, vol. 57, no. 4, pp. 527-544, 2000.

[28] J. Wurman, "Deployments of a 12-site in situ Wind/T/RH instrument array in tornadoes," in Proceedings of the 24th Conference on Severe Local Storms, American Meteorological Society, Savannah, Ga, USA, 2008. 

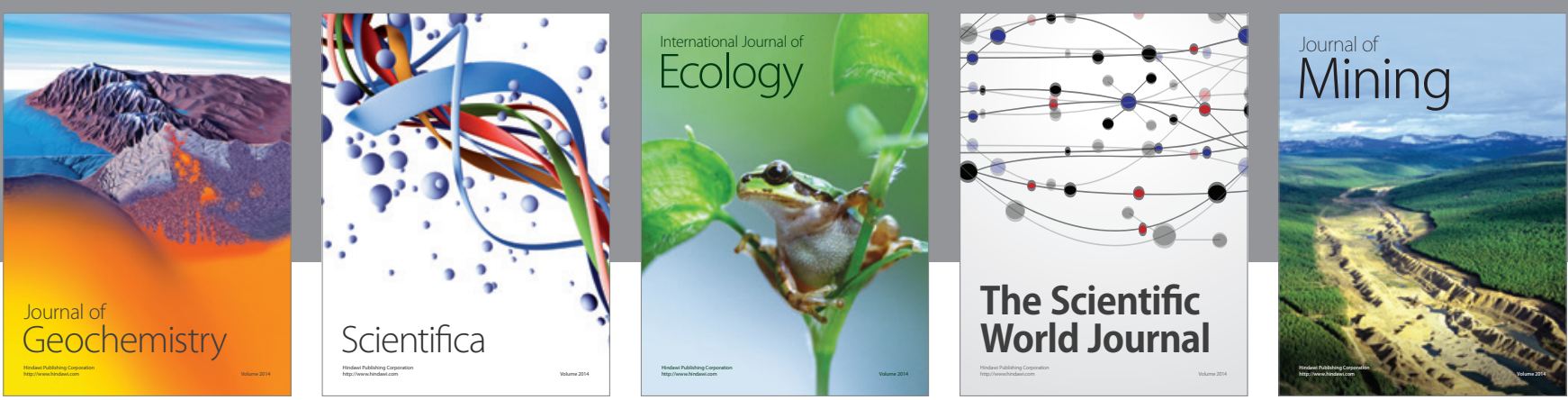

The Scientific World Journal
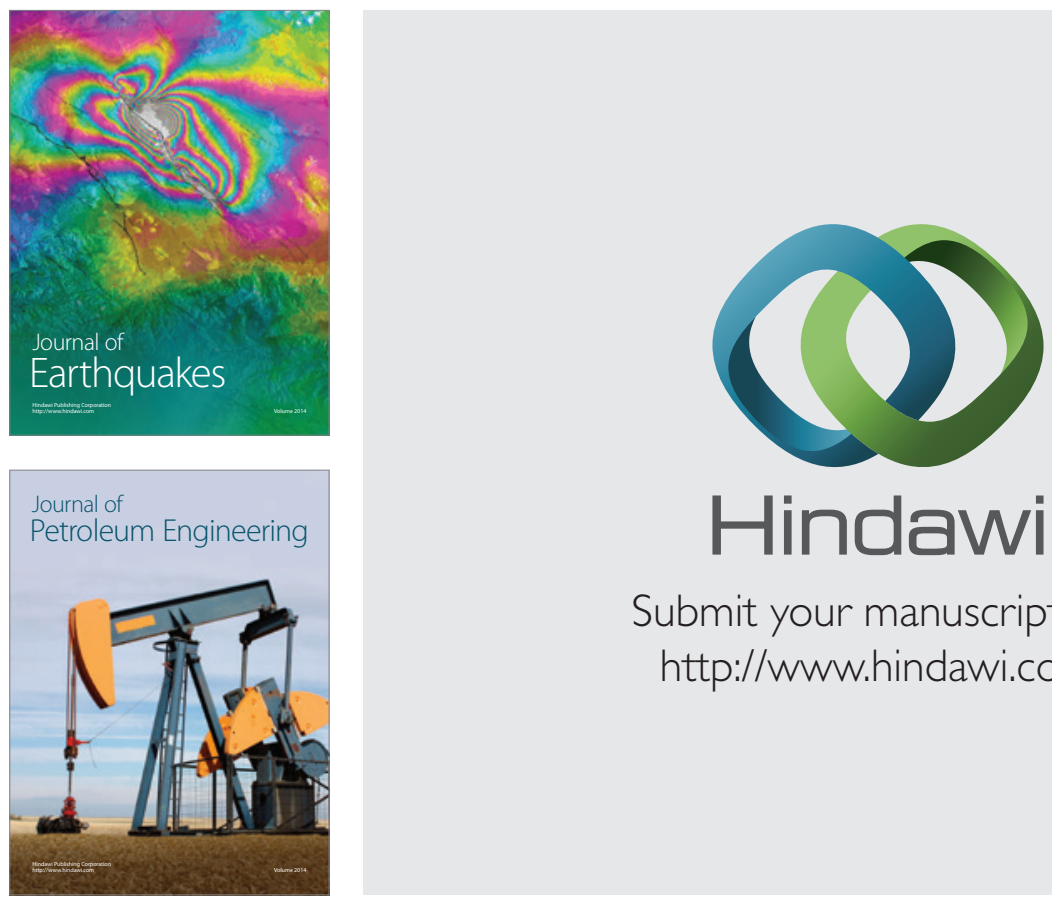

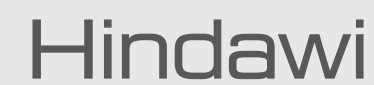

Submit your manuscripts at

http://www.hindawi.com
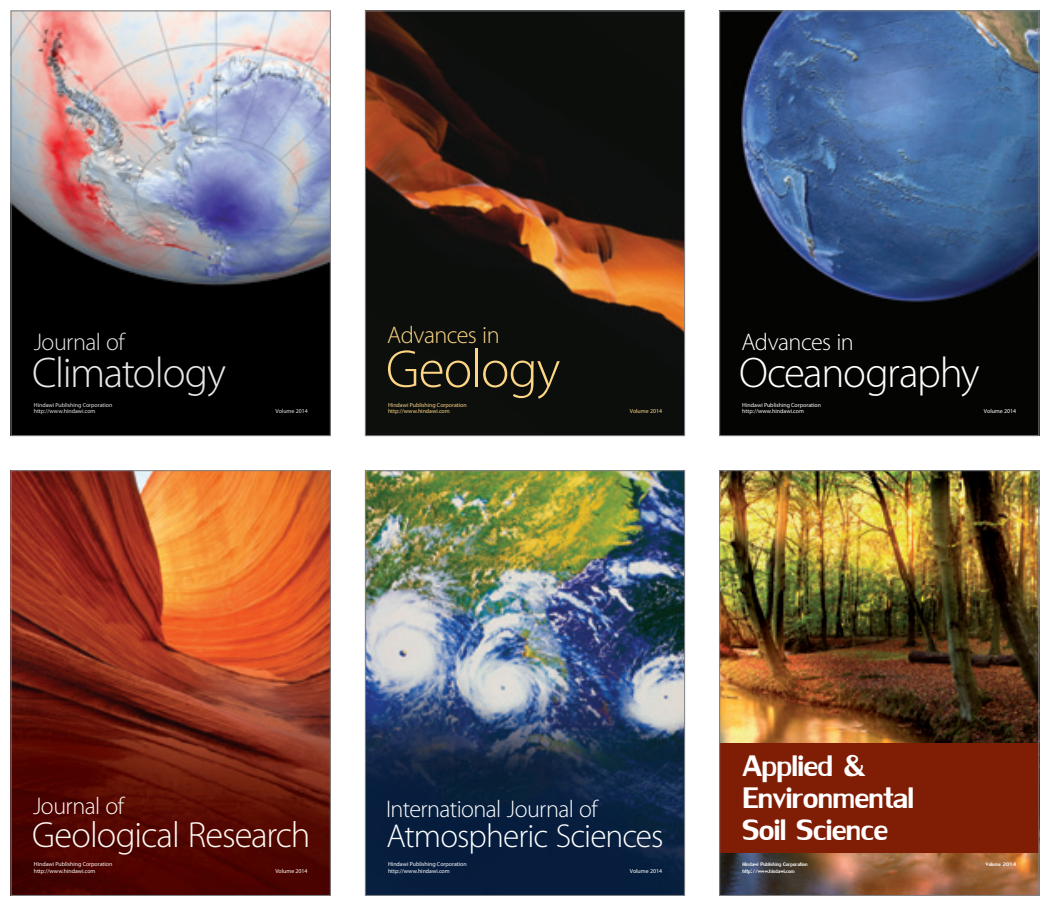
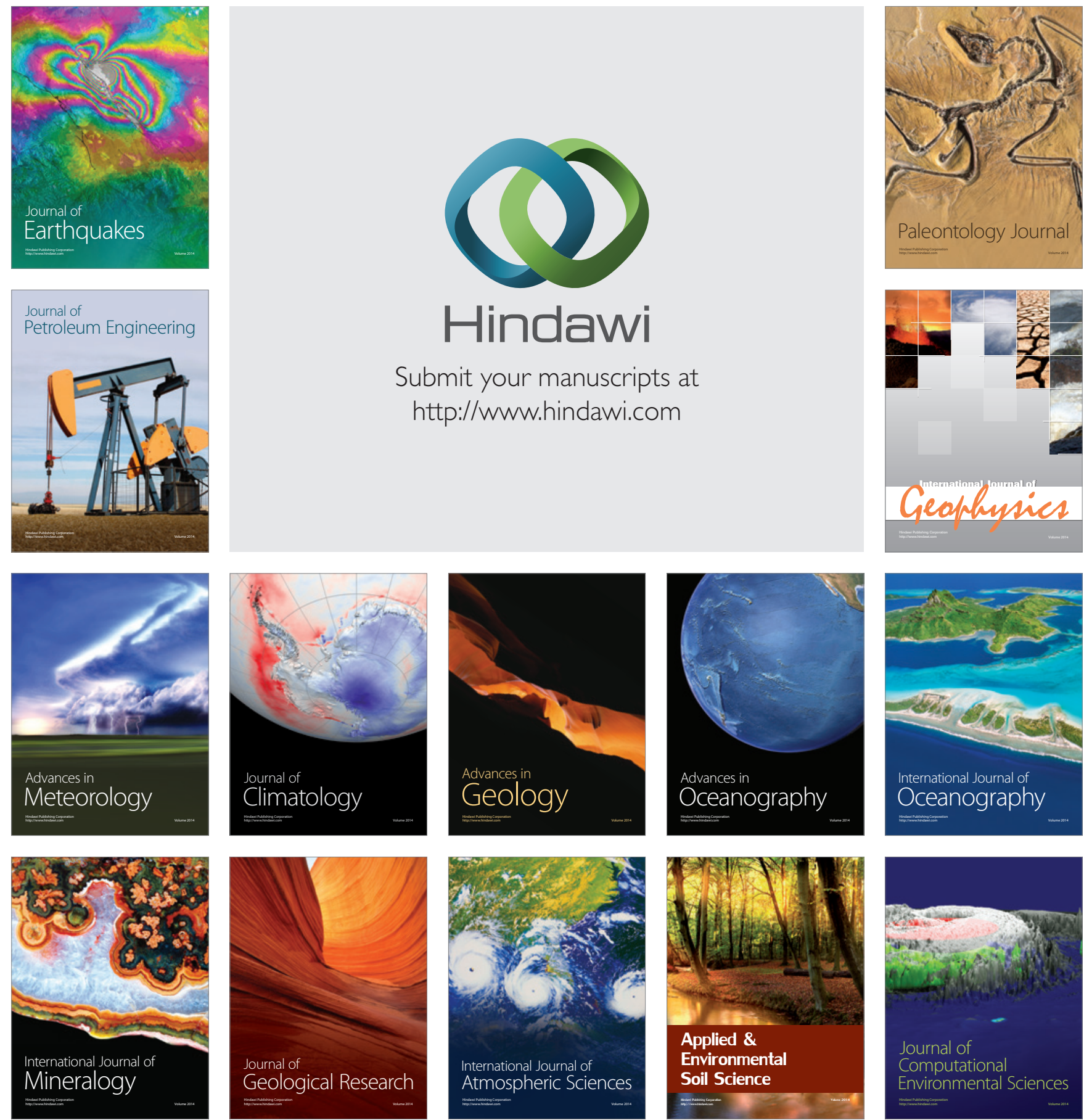